\title{
Supporting Information \\ PCAViz: An Open-Source Python/JavaScript Toolkit for Visualizing Molecular Dynamics Simulations in the Web Browser
}

Sayuri Pacheco1,t, Jesse C. Kaminsky1,t, lurii K. Kochnev1, Jacob D. Durrant1, ${ }^{*}$

1 Department of Biological Sciences, University of Pittsburgh, Pittsburgh, Pennsylvania, 15260, United States

† The authors wish it to be known that, in their opinion, the first two authors should be regarded as joint First Authors.

*Email: durrantj@pitt.edu 
Table S1. PCAViz accuracy when applied to a LARP1 simulation. Using various cumulative-variance and rounding-precision parameters, we compressed a 100-frame simulation of LARP1 (1,326 nonhydrogen atoms) with the PCAViz Compressor. We then decompressed each simulation to recover the atomic Cartesian coordinates. To judge PCAViz accuracy, we calculated the average RMSD (plus or minus the standard deviation) between each frame of the original trajectory and the corresponding frame of the PCAViz-processed trajectory. We also include the file size of each output JSON file so users can judge the extent of compression. The number of top components that PCAViz retained in order to account for the user-specified cumulative-variance cutoff is given in parentheses.

\begin{tabular}{|c|c|c|c|}
\hline $\begin{array}{l}\text { Cumulative-Variance Cutoff } \\
\text { (Number of Components) }\end{array}$ & $\begin{array}{l}\text { Precision: } \\
\text { Tenth }\end{array}$ & $\begin{array}{l}\text { Precision: } \\
\text { Hundredth }\end{array}$ & $\begin{array}{l}\text { Precision: } \\
\text { Thousandth }\end{array}$ \\
\hline $20 \%(1)$ & $\begin{array}{c}1.55 \AA \pm 0.18 \\
48 \mathrm{~K}\end{array}$ & $\begin{array}{c}1.47 \AA \pm 0.18 \\
56 \mathrm{~K}\end{array}$ & $\begin{array}{c}1.46 \AA \pm 0.18 \\
65 \mathrm{~K}\end{array}$ \\
\hline $25 \%(1)$ & $\begin{array}{c}1.55 \AA \pm 0.18 \\
48 \mathrm{~K}\end{array}$ & $\begin{array}{c}1.47 \AA \pm 0.18 \\
56 \mathrm{~K}\end{array}$ & $\begin{array}{c}1.46 \AA \pm 0.18 \\
65 \mathrm{~K}\end{array}$ \\
\hline $30 \%(2)$ & $\begin{array}{c}1.54 \AA \pm 0.17 \\
56 \mathrm{~K}\end{array}$ & $\begin{array}{c}1.38 \AA \pm 0.14 \\
66 \mathrm{~K}\end{array}$ & $\begin{array}{c}1.37 \AA \pm 0.14 \\
78 \mathrm{~K}\end{array}$ \\
\hline $40 \%(3)$ & $\begin{array}{c}1.52 \AA \pm 0.17 \\
64 \mathrm{~K}\end{array}$ & $\begin{array}{c}1.32 \AA \pm 0.12 \\
75 \mathrm{~K}\end{array}$ & 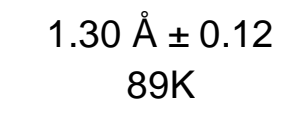 \\
\hline $50 \%(5)$ & $\begin{array}{c}1.49 \AA \pm 0.16 \\
81 \mathrm{~K}\end{array}$ & $\begin{array}{c}1.23 \AA \pm 0.10 \\
94 \mathrm{~K}\end{array}$ & $\begin{array}{c}1.20 \AA \pm 0.10 \\
111 \mathrm{~K}\end{array}$ \\
\hline $60 \%(9)$ & $\begin{array}{c}1.45 \AA \pm 0.16 \\
113 \mathrm{~K}\end{array}$ & $\begin{array}{c}1.10 \AA \pm 0.080 \\
131 \mathrm{~K}\end{array}$ & $\begin{array}{c}1.08 \AA \pm 0.080 \\
157 \mathrm{~K}\end{array}$ \\
\hline $70 \%(15)$ & $\begin{array}{c}1.42 \AA \pm 0.16 \\
162 \mathrm{~K}\end{array}$ & $\begin{array}{c}0.99 \AA \pm 0.054 \\
188 \mathrm{~K}\end{array}$ & $\begin{array}{c}0.95 \AA \pm 0.056 \\
227 \mathrm{~K}\end{array}$ \\
\hline $75 \%(20)$ & $\begin{array}{c}1.41 \AA \pm 0.16 \\
203 \mathrm{~K}\end{array}$ & $\begin{array}{c}0.91 \AA \pm 0.049 \\
235 \mathrm{~K}\end{array}$ & $\begin{array}{c}0.87 \AA \pm 0.052 \\
285 \mathrm{~K}\end{array}$ \\
\hline $80 \%(27)$ & $\begin{array}{c}1.39 \AA \pm 0.16 \\
260 \mathrm{~K}\end{array}$ & $\begin{array}{c}0.82 \AA \pm 0.040 \\
300 \mathrm{~K}\end{array}$ & $\begin{array}{c}0.77 \AA \pm 0.044 \\
365 \mathrm{~K}\end{array}$ \\
\hline $90 \%(48)$ & $\begin{array}{c}1.36 \AA \pm 0.16 \\
431 \mathrm{~K}\end{array}$ & $\begin{array}{c}0.62 \AA \pm 0.038 \\
497 \mathrm{~K}\end{array}$ & $\begin{array}{c}0.55 \AA \pm 0.046 \\
608 \mathrm{~K}\end{array}$ \\
\hline $100 \%(99)$ & $\begin{array}{c}1.34 \AA \pm 0.16 \\
845 \mathrm{~K}\end{array}$ & $\begin{array}{c}0.31 \AA \pm 0.033 \\
978 \mathrm{~K}\end{array}$ & $\begin{array}{c}0.032 \AA \pm 0.0035 \\
1.2 \mathrm{M}\end{array}$ \\
\hline
\end{tabular}


Table S2. PCAViz accuracy when applied to a TEM-1 simulation. Using various cumulative-variance and rounding-precision parameters, we compressed a 1,000-frame simulation of TEM- $1 \beta$-lactamase (2,030 non-hydrogen atoms) with the PCAViz Compressor. We then decompressed each simulation to recover the atomic Cartesian coordinates. To judge PCAViz accuracy, we calculated the average RMSD (plus or minus the standard deviation) between each frame of the original trajectory and the corresponding frame of the PCAViz-processed trajectory. We also include the file size of each output JSON file so users can judge the extent of compression. The number of top components that PCAViz retained in order to account for the user-specified cumulative-variance cutoff is given in parentheses.

\begin{tabular}{|c|c|c|c|}
\hline $\begin{array}{l}\text { Cumulative-Variance Cutoff } \\
\text { (Number of Components) }\end{array}$ & $\begin{array}{l}\text { Precision: } \\
\text { Tenth }\end{array}$ & $\begin{array}{l}\text { Precision: } \\
\text { Hundredth }\end{array}$ & $\begin{array}{l}\text { Precision: } \\
\text { Thousandth }\end{array}$ \\
\hline $20 \%(2)$ & $\begin{array}{c}0.92 \AA \pm 0.068 \\
94 \mathrm{~K}\end{array}$ & $\begin{array}{c}0.85 \AA \pm 0.056 \\
111 \mathrm{~K}\end{array}$ & $\begin{array}{c}0.85 \AA \pm 0.056 \\
131 \mathrm{~K}\end{array}$ \\
\hline $25 \%(4)$ & $\begin{array}{c}0.92 \AA \pm 0.068 \\
125 \mathrm{~K}\end{array}$ & $\begin{array}{c}0.82 \AA \pm 0.053 \\
147 \mathrm{~K}\end{array}$ & $\begin{array}{c}0.81 \AA \pm 0.053 \\
174 \mathrm{~K}\end{array}$ \\
\hline $30 \%(5)$ & $\begin{array}{c}0.91 \AA \pm 0.069 \\
141 \mathrm{~K}\end{array}$ & $\begin{array}{c}0.80 \AA \pm 0.052 \\
165 \mathrm{~K}\end{array}$ & $\begin{array}{c}0.79 \AA \pm 0.052 \\
196 \mathrm{~K}\end{array}$ \\
\hline $40 \%(10)$ & $\begin{array}{c}0.90 \AA \pm 0.070 \\
217 \mathrm{~K}\end{array}$ & $\begin{array}{c}0.75 \AA \pm 0.047 \\
254 \mathrm{~K}\end{array}$ & $\begin{array}{c}0.74 \AA \pm 0.048 \\
303 \mathrm{~K}\end{array}$ \\
\hline $50 \%(19)$ & $\begin{array}{c}0.89 \AA \pm 0.070 \\
354 \mathrm{~K}\end{array}$ & $\begin{array}{c}0.69 \AA \pm 0.037 \\
412 \mathrm{~K}\end{array}$ & $\begin{array}{c}0.67 \AA \pm 0.038 \\
497 \mathrm{~K}\end{array}$ \\
\hline $60 \%(34)$ & $\begin{array}{c}0.88 \AA \pm 0.071 \\
581 \mathrm{~K}\end{array}$ & $\begin{array}{c}0.62 \AA \pm 0.031 \\
676 \mathrm{~K}\end{array}$ & $\begin{array}{c}0.60 \AA \pm 0.031 \\
818 \mathrm{~K}\end{array}$ \\
\hline $70 \%(61)$ & $\begin{array}{c}0.86 \AA \pm 0.072 \\
988 \mathrm{~K}\end{array}$ & $\begin{array}{c}0.55 \AA \pm 0.023 \\
1.2 \mathrm{M}\end{array}$ & $\begin{array}{c}0.52 \AA \pm 0.023 \\
1.4 \mathrm{M}\end{array}$ \\
\hline $75 \%(84)$ & $\begin{array}{c}0.86 \AA \pm 0.072 \\
1.4 \mathrm{M}\end{array}$ & $\begin{array}{c}0.51 \AA \pm 0.020 \\
1.6 \mathrm{M}\end{array}$ & $\begin{array}{c}0.48 \AA \pm 0.019 \\
1.9 \mathrm{M}\end{array}$ \\
\hline $80 \%(115)$ & $\begin{array}{c}0.86 \AA \pm 0.073 \\
1.8 \mathrm{M}\end{array}$ & $\begin{array}{c}0.47 \AA \pm 0.017 \\
2.1 \mathrm{M}\end{array}$ & $\begin{array}{c}0.43 \AA \pm 0.016 \\
2.5 \mathrm{M}\end{array}$ \\
\hline $90 \%(240)$ & $\begin{array}{c}0.85 \AA \pm 0.073 \\
3.6 \mathrm{M}\end{array}$ & $\begin{array}{c}0.36 \AA \pm 0.013 \\
4.2 \mathrm{M}\end{array}$ & $\begin{array}{c}0.30 \AA \pm 0.0089 \\
5.2 \mathrm{M}\end{array}$ \\
\hline $100 \%(1000)$ & $\begin{array}{c}0.84 \AA \pm 0.074 \\
15 \mathrm{M}\end{array}$ & $\begin{array}{c}0.21 \AA \pm 0.016 \\
17 \mathrm{M}\end{array}$ & $\begin{array}{c}0.021 \AA \pm 0.0017 \\
21 \mathrm{M}\end{array}$ \\
\hline
\end{tabular}

